Yinghua Qi, Akash Nathani, Jianxin Zhang, Zhengji Song, Chandra Shekhar Sharma and Sunil K. Varshney*

\title{
Synthesis of amphiphilic poly(ethylene glycol)-block-poly(methyl methacrylate) containing trityl ether acid cleavable junction group and its self-assembly into ordered nanoporous thin films
}

https://doi.org/10.1515/epoly-2020-0013

Received October 02, 2019; accepted November 25, 2019.

\begin{abstract}
A strategy for the synthesis of well defined poly(ethylene glycol)-block-poly(methyl methacrylate) diblock copolymers containing trityl ether acid cleavable junctions is demonstrated. This approach is achieved by using a combination of poly(ethylene glycol) macroinitiator containing a trityl ether end group, which is susceptible to acid cleavage, and atom transfer radical polymerization technique. The trityl ether linkage between blocks can be readily cleaved in solution or in solid phase under very mild acid condition, which has been confirmed by ${ }^{1} \mathrm{H}$ NMR. These diblock copolymers have been used to successfully fabricate nanoporous thin films by acid cleavage of trityl ether junction followed by complete removal of poly(ethylene glycol) block. The fabricated nanoporous thin films may have a wide range of application such as Recessed Nanodisk-array electrode (RNE) or as a template to fabricate nanoelectrode array for senor applications.
\end{abstract}

Keywords: amphiphilic block copolymer; acid cleavable; trityl ether group; poly(methyl methacrylate); nanoporous thin film

\footnotetext{
*Corresponding author: Sunil K. Varshney, Polymer Source Inc., 124 Avro Street, Dorval (Montreal), Quebec H9P 2X8, Canada, email: info@polymersource.com

Yinghua Qi, Jianxin Zhang, Zhengji Song and Sunil K. Varshney, Polymer Source Inc., 124 Avro Street, Dorval (Montreal), Quebec H9P 2X8, Canada

Akash Nathani, Chandra Shekhar Sharma, Creative \& Advanced Research Based On Nanomaterials (CARBON) Lab, Department of Chemical engineering, Indian Institute of Technology, Hyderabad, Kandi-502285, Telangana, India
}

\section{Introduction}

Highly ordered nanoporous thin films have attracted continuous attention because they have found numerous applications in the areas of separation materials (1), gas storage materials $(2,3)$, drug delivery systems (4-7), photonic band gap materials (8), nanoreactors (9). Among the strategies to produce nanoporous structures, self-assembly of block copolymers (BCPs), especially amphiphilic BCPs, have been proved to be an efficient way to fabricate nanoporous thin films by selective removal of minority domain. The size of nanoporous structure can be precisely tuned by the block copolymer compositions, molecular weights and self assembly conditions (10). However, selective removal of one domain from block copolymers remains a challenging problem. Until now, diverse methods have been employed for the selective removal of minority components to create nanopores, including chemical etching, reactive ion etching and UV degradation (11-13). However, most of these methods require harsh conditions, and are limited to certain degradable polymers. To overcome these drawbacks, the introduction of a cleavable linkage between two blocks has been proposed and developed (14-18), by which the block copolymers can be cleaved rapidly and efficiently under very mild conditions. The cleavage of the junction point of block copolymers in the ordered state followed by removal of the minority block has been proven to be a very useful strategy for the production of nanoporous structures.

Venkataraman et al. $(19,20)$ and Hawker et al. (21) have reported the synthesis of block copolymers having acid cleavable junctions based on triphenylmethyl (trityl) ether group and demonstrated that highly ordered nanoporous thin films had been fabricated with controlled porosity. It is well known that trityl ether groups are stable to strong 
nucleophiles, bases and radicals and thus are inert to radical and anionic polymerization conditions that are commonly used in polymer synthesis. But upon acidic treatment, trityl ethers can readily generate trityl cations. The positive charge on the $\alpha$-carbon atom is stabilized by the resonance effect of three aromatic rings and is further stabilized by introducing substituents that can facilitate the charge distribution, e.g. ortho- or para- alkyl or alkoxy groups. This particular property makes trityl ethers acid-labile and can be readily cleaved by Bronsted or Lewis acids at ambient conditions (22). As a result, trityl ether linker appears to be a very attractive linker between the respective blocks of multiblock polymers for the development of highly ordered porous materials. Up to date, the BCPs containing trityl ether cleavable group at junctions that have been developed are mostly based on polystyrene block. In our study, we chose to focus on trityl ether-containing copolymers based on poly(methyl methacrylate) (PMMA) block, which is one of the most important building blocks used in amphiphilic block copolymers, and unique properties of ordered nanoporous thin films are expected from these novel materials.

In the preparation of previously reported trityl ether-containing copolymers, a moiety bearing 2,2,6,6-tetramethylpiperidine- $\mathrm{N}$-oxyl (TEMPO) was commonly incorporated at the chain end of first polymer block via a trityl ether linker, and nitroxide-mediated radical polymerizations (NMP) was therefore used to initiate the second blocks, e.g. polystyrene blocks (19-21). It is known that NMP is a versatile and easy polymerization technique to carry out and can work well with styrenic type of monomers. While NMP has been used with great success in the preparation of a wide variety of interesting polymer structures, it has two major disadvantages: [1] slow polymerization kinetics that requires high temperatures and lengthy polymerization times; [2] inability to polymerize methacrylate type of monomers in a controlled fashion due to side reactions and/or slow recombination of the polymer radical with nitroxide. These inherent problems have prevented NMP technique from its widespread applications, especially in the preparation of block copolymers based on methacrylate monomers (23). Therefore, there is a need to explore a novel synthetic approach capable to provide easy access to trityl ethercontaining BCPs based on polymethacrlyate blocks in a controlled manner. Nowadays, ATRP has been recognized as one of the most useful approaches towards diverse block copolymer systems in terms of simplicity and monomer compatibility. It allows a broader range of monomers to be polymerized with the same initiation system compared to NMP initiating system. Specifically, ATRP is preferable to
NMP method for the preparation of methacrylate type of polymers, such as PMMA. Therefore, ATRP technique was employed in our study, and two BCPs using PMMA as the majority blocks and poly(ethylene glycol)s (PEG)s as the minority blocks have been successfully prepared with well defined polymer structures.

However, in order to achieve the complete removal of minority block, a high degree of phase segregation is necessary. The BCP films obtained by spin coating with fast evaporating solvent would cause vitrification, which leads to the non-equilibrium morphology lacking longrange order (24). To assist the microphase separation, it is necessary to impart sufficient chain mobility. Different annealing conditions have been used to impart mobility to the polymer chains. During annealing, the defects in the film, usually in the form of dislocations, which behaves like the quasi-particles diffuses through the film. This coarsening leads to a decrease in the defect density and an increase in the grain size $(\xi)$ that scales as, $\xi=\mathrm{kt}^{\alpha}$. Here the exponent $\alpha$ describes the mechanism and the pre-factor $\mathrm{k}$ amounts for the scale of kinetics, including the role of temperature (25). Thermal annealing, which involves the vacuum heating above the glass transition temperature $\left(\mathrm{T}_{\mathrm{g}}\right)$, is the most widely used technique to enhance ordering kinetics. However, thermal annealing is not a suitable choice if there is crystallization or degradation of one or more polymeric blocks [26]. Also, for the high molecular weight polymers, the diffusion is too slow to achieve any macroscopic grain size over the reasonable time scale (27). Another technique used to improve ordering involves the use of plasticizers during (28) or after film casting such as solvent vapour annealing (SVA) and direct immersion annealing (DIA). In solvent vapour annealing, the film is exposed to vapours (mostly saturated) of a liquid which is a good solvent for either one or more than one blocks. The solvent uptake causes swelling and plasticization of the film, thus improving ordering. However, the overswelling or differential swelling may lead to dewetting and topographic rearrangement $(29,30)$. Moreover, the SVA films require further thermal annealing to remove the wrinkles formed due to buckling during the deswelling (31). DIA, which is a more facile way involves the complete immersion of the film in a mixture of a poor or non-solvent and a good solvent for one or more than blocks. Here the non-solvent controls the rate of film dissolution and the good solvent imparts the necessary plasticization. Modi et al. (32) have demonstrated the use of DIA for ordering PS-b-PMMA thin films. A significant enhancement in the grain growth kinetics was observed when compared to thermal annealing as indicated by the pre-factor k. They have also shown that although the ordered morphology 
was rapidly obtained after immersion, the morphology was stable under the extended immersion time, and the same was in accord with the predicted equilibrium morphology (32). Park et al. used the DIA along with the nanolithography patterns for the ordering of PS-b-PDMA (33) whereas Longanecker et al. used DIA for the ordering of highly loaded nano filled PS-b-PMMA (34).

In this paper, we present an ATRP method with the use of a trityl ether end functionalized poly(ethylene glycol) (PEG) as macroinitiator to synthesize cleavable BCPs based on PMMA. The thin films obtained by coating the dilute solution of synthesized BCP were annealed using DIA technique to assist phase separation followed by the acid vapor treatment to generate the excess porosity.

\section{Experimental section}

\subsection{Materials}

THF was purified by refluxing over fresh sodium benzophenone complex and distilling before the use. Methyl methacrylate (MMA) and anisole were purchased from Sigma- Aldrich and distilled over $\mathrm{CaH}_{2}$ prior to the use. 4-Vinylphenyl diphenyl methanol was prepared from bromostyrene and benzophenone via Grignard reaction in THF with a yield of $62 \%$. Monomethoxy PEG $(5000 \mathrm{~g} / \mathrm{mol})$ was prepared by anionic polymerization of ethylene oxide in THF at $30^{\circ} \mathrm{C}$ followed by termination with dilute $\mathrm{HCl}$. $\alpha$-Methoxy- $\omega$-mesylate end functionalized poly(ethylene glycol) (MPEG-mesylate) was prepared using monomethoxy PEG (5000 g/mol) according to literature method (35). All the other chemicals and solvents were purchased from Sigma-Aldrich and used without further purification.

\subsection{Characterization method}

${ }^{1} \mathrm{H}$ NMR spectra were performed on a Bruker DRX spectrometer $(500 \mathrm{MHz})$ or Oxford AS400 spectrometer (400 MHz). The molecular weight and polydispersity index of polymers were determined by size exclusion chromatography (SEC) with tri-detector system equipped with a refractive index, viscometer and multi angle light scattering detectors using DMF containing $0.023 \mathrm{M}$ of $\mathrm{LiBr}$ as mobile phase $\left(\right.$ at $50^{\circ} \mathrm{C}$ and a flow rate of $0.7 \mathrm{~mL} / \mathrm{min}$ ). FTIR were recorded on a PerkinElmer FTIR Spectrum Two by casting the polymer film on a $\mathrm{KBr}$ disk. Differential scanning calorimetry (DSC) measurements were performed on a TA Instruments Q100 with a scan rate of $10^{\circ} \mathrm{C} / \mathrm{min}$ under nitrogen. The Atomic force microscopy (AFM) data was obtained using Bruker Dimension with Silicon etched probe having spring constant $3 \mathrm{~N} / \mathrm{m}$ and operated in intermittent contact (Tapping) mode.

\subsection{Synthesis of vinyl trityl ether-end functionalized PEG (PEG-VinylTTE)}

To a $500 \mathrm{~mL}$ flask containing THF (120 mL) was charged sodium hydride (60\% suspension in mineral oil) $(7 \mathrm{~g}$, $0.175 \mathrm{~mol}$ ) and 4-vinylphenyl diphenyl methanol (10 g, $0.035 \mathrm{~mol}$ ). After the suspension was refluxed for $24 \mathrm{~h}$ and then cooled down to room temperature, it was transferred into a solution of MPEG-mesylate $\left(\mathrm{M}_{\mathrm{n}}=5000 \mathrm{~g} / \mathrm{mol}\right)$ (120 g, $0.024 \mathrm{~mol})$ in THF $(500 \mathrm{~mL})$ by a capillary. The solution was stirred at reflux temperature for another $24 \mathrm{~h}$ and then allowed to cool to room temperature. After the mixture was filtered through a filter paper and concentrated under vacuum, the product was precipitated into cold diethyl ether and reprecipitated one more time. The resulting white powder was collected by filtration and dried under vacuum at $45^{\circ} \mathrm{C}$ for $12 \mathrm{~h}$ (90\% yield). ${ }^{1} \mathrm{H}$ NMR (400 MHz, $\mathrm{CDCl}_{3}$ ): 7.18-7.48 (m, 14H), $6.69(\mathrm{dd}, J=8.0 \mathrm{~Hz}$, $12.0 \mathrm{~Hz}, 1 \mathrm{H}), 5.69(\mathrm{~d}, J=12.0 \mathrm{~Hz}, 1 \mathrm{H}), 5.20(\mathrm{~d}, J=12.0 \mathrm{~Hz}$, $1 \mathrm{H}), 3.64(\mathrm{~m}, 452 \mathrm{H}), 3.37(\mathrm{~s}, 3 \mathrm{H})$.

\subsection{Synthesis of hydroxy ethyl trityl ether-end functionalized PEG (PEG-HydroxyTTE)}

To a $1 \mathrm{~L}$ flask containing THF $(500 \mathrm{~mL})$ was charged polymer PEG-VinylTTE $\left(M_{n}=5000 \mathrm{~g} / \mathrm{mol}, 30 \mathrm{~g}, 6 \mathrm{mmol}\right)$. Then 9-Borabicyclo[3.3.1]nonane (9-BBN) solution in THF (0.5 M, $60 \mathrm{~mL}, 0.030 \mathrm{mmol}$ ) was dropwisely added into polymer solution at room temperature and the mixture was stirred for $24 \mathrm{~h}$. Then sodium hydroxide solution (3 $\mathrm{N}, 15 \mathrm{~mL}, 0.045 \mathrm{~mol}$ ) and hydrogen peroxide solution $(50 \mathrm{w} / \mathrm{w} \%, 10 \mathrm{~mL}, 0.145 \mathrm{~mol})$ were sequentially added into the reaction mixture. The resulting solution was stirred at $40^{\circ} \mathrm{C}$ for $12 \mathrm{~h}$ to get clear solution. After the polymer solution was concentrated under vacuum, the product was extracted with dichloromethane and water. The organic phase was washed with water two time and the combined organic phase was dried over $\mathrm{MgSO}_{4}$ and was filtered through a filter paper. Finally the polymer solution was precipitated into cold diethyl ether and reprecipitated one more time to provide PEG-HydroxyTTE as a white solid product. (82\% yield). ${ }^{1} \mathrm{H}$ NMR (500 $\mathrm{MHz}, \mathrm{CDCl}_{3}$ ): 7.18-7.48 (m, 14H), 3.64 (m, 454H), 3.37 (s, 3H). 


\subsection{Synthesis of 2-bromo isobutyryl ethyl trityl ether-end functionalized PEG (PEG-TTE-Br)}

To a $1 \mathrm{~L}$ flask containing THF (500 mL) equipped with a three-way stopcock bearing rubber septum was charged OH-terminated polyethylene glycol PEG-HydroxyTTE ( $25 \mathrm{~g}, 5 \mathrm{mmol}$ ) and the flask was purged with argon. Then triethyl amine (1.1 g, $10.6 \mathrm{mmol}$ ) and 2-bromoisobutyryl bromide ( $2.5 \mathrm{~g}, 10.8 \mathrm{mmol}$ ) were sequentially added via a syringe at room temperature. The reaction mixture was stirred at room temperature for $12 \mathrm{~h}$ and then filtered through a filter paper. The filtrate was concentrated under vacuum and precipitated into cold diethyl ether. The final product was purified by dissolving in chloroform and reprecipitating into cold diethyl ether 2 times ( $90 \%$ yield). ${ }^{1} \mathrm{H}$ NMR (500 MHz, $\mathrm{CDCl}_{3}$ ): 7.18-7.48 (m, 11H), 4.29 (t, 2H), 3.64 (m, 456H), 3.34 (s, 3H), 1.90 (s, 6H), 1.89 (s, 15H).

\subsection{Synthesis of acid cleavable poly(ethylene glycol)-block-poly(methyl methacrylate) copolymers (PEG-b-PMMA)}

Synthesis of PEG- $b$-PMMA (Polymer $1, M_{n}=5,000-b$ $55,000 \mathrm{~g} / \mathrm{mol}$ ) is illustrated below as an example.

\subsubsection{Synthesis of PEG-b-PMMA (Polymer 1 , $M_{n}=5,000-b-55,000 \mathrm{~g} / \mathrm{mol}$ )}

PEG-TTE-Br $\left(M_{n}=5,000 \mathrm{~g} / \mathrm{mol} ; 1.0 \mathrm{~g}, 0.2 \mathrm{mmol}\right)$, MMA (15 g), copper chloride (I) $(20 \mathrm{mg}, 0.2 \mathrm{mmol}$ ), hexamethylenetetramine ( $28 \mathrm{mg}, 0.2 \mathrm{mmol}$ ) were charged into a Schlenk flask containing anisole $(15 \mathrm{~mL})$. The reaction mixture was degassed by three freeze-pumpthaw cycles and the flask was finally sealed under vacuum. The reaction solution was stirred at $80^{\circ} \mathrm{C}$ for $12 \mathrm{~h}$. After cooling down, the reaction solution was filtered through a short pad of neutral alumina and then precipitated into hexanes. The crude diblock copolymer was dissolved again in the THF and reprecipitated into hexanes. The final product was dried under vacuum at $50^{\circ} \mathrm{C}$ for $12 \mathrm{~h} .{ }^{1} \mathrm{H}$ NMR (400 MHz, $\mathrm{CDCl}_{3}$ ): 3.64 (br, 454H), 3.59 (br, 1653H), 1.70-2.10 (m, 962H), 1.20 (br, 70H), 1.01 (br, 587H), 0.84 (br, 993H).

\subsubsection{Synthesis of PEG- $b$-PMMA (Polymer 2, $M_{\mathrm{n}}=5,000-b-85,000 \mathrm{~g} / \mathrm{mol}$ )}

${ }^{1} \mathrm{H} \mathrm{NMR} \mathrm{(400} \mathrm{MHz,} \mathrm{CDCl}_{3}$ ): 3.64 (br, 454H), 3.59 (br, 2560H), 1.70-2.10 (m, 1619H), 1.20 (br, 163H), 1.01 (br, 1003H), 0.84 (br, 1406H).

\subsection{Acid cleavage of PEG-b-PMMA diblock copolymer in solution}

The acid cleavage of Polymer 2 (PEG- $b$-PMMA, $\mathrm{M}_{\mathrm{n}}=5,000$ $b-85,000 \mathrm{~g} / \mathrm{mol}$ ) was illustrated as an example. Polymer 2 $(50 \mathrm{mg}$ ) was dissolved in toluene $(5 \mathrm{~mL})$. To this solution was added trifluoroacetic acid (TFA, $0.5 \mathrm{~g}$ ) at room temperature and the color of the solution turned to orange right away. After the solution was stirred for $15 \mathrm{~min}$, the toluene and excess TFA were removed under vacuum. The residue was dissolved in chloroform $(5 \mathrm{~mL})$ and washed with water three times. The chloroform layer was combined and chloroform was removed under vacuum to recover product.

\subsection{Fabrication of mesoporous thin films}

Polymers 1 and 2 were used to demonstrate the phase separation behavior and the efficiency of the acid cleavage. To fabricate the thin film, $0.7 \%(\mathrm{w} / \mathrm{v})$ solution of the polymer in toluene was spin-coated on $\mathrm{Si}\left(\begin{array}{lll}1 & 0 & 0\end{array}\right)$ wafer at $3000 \mathrm{rpm}$ for $30 \mathrm{~s}$. Prior to coating, the wafer was cleaned by sonicating in isopropyl alcohol and washing with DI water followed by cleaning with oxygen plasma treatment for $15 \mathrm{~min}$. The as-coated thin film was treated with the mixture of toluene and $\mathrm{n}$-heptane with the mole fraction of the toluene, $\mathrm{xt}=0.57$ to carry out the DIA (32). The films were then washed with $n$-heptane to remove any residual solvent and dried on a hotplate at $60^{\circ} \mathrm{C}$. Later the films were exposed to the saturated vapours of TFA for 30s followed by washing with a mixture of methanol/DI water (1:1).

\section{Results and discussion}

The unique features of trityl ether group makes it a very useful and versatile linker between the components of multiblock copolymers. In order to introduce such acid cleavable group into the junction of PEG- $b$-PMMA block copolymers, a novel end functionalized ATRP PEG-macroinitiator containing trityl ether group (PEGTTE-Br) was designed and prepared. The synthetic route is presented in Scheme 1.

The trityl ether end functionalized ATRP macroinitiator was prepared via a three step reactions with a satisfactory total yield starting from etherification of $\alpha$-methoxy- $\omega$-mesylate poly(ethylene oxide) with 4-vinylphenyl diphenyl methanol. The disappearance of the mesylate methyl peak at $3.06 \mathrm{ppm}$ and appearance of vinyl peak between $5.20-6.70 \mathrm{ppm}$ indicated the reaction 


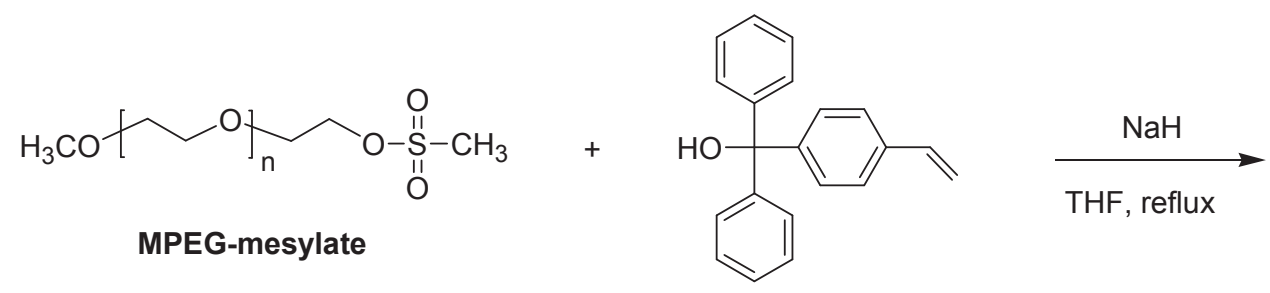

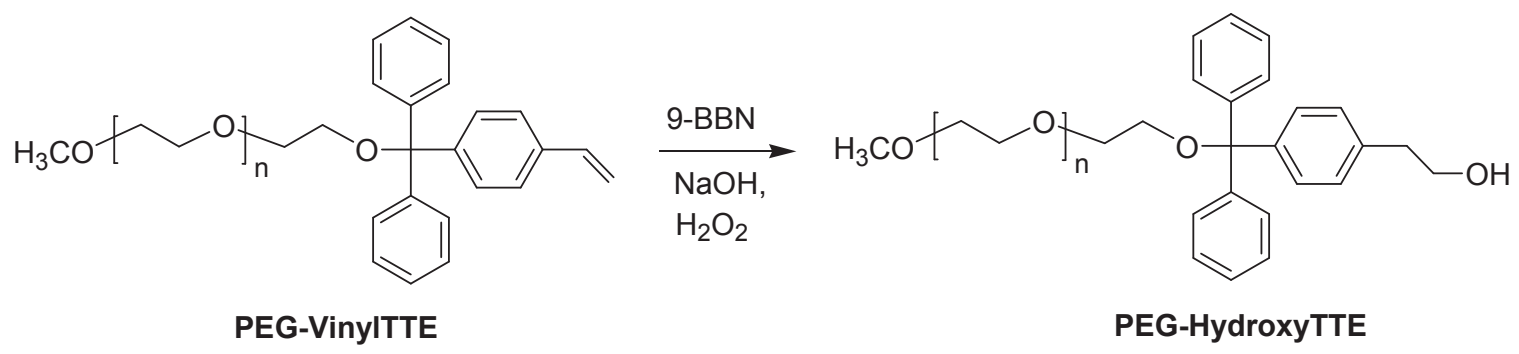<smiles>COC(COCCOC(c1ccccc1)(c1ccccc1)c1ccc(CCOC(=O)C(C)(C)Br)cc1)C(F)F</smiles>

Scheme 1: Synthesis of ATRP macroinitiator PEG-TTE-Br.

was complete. Herein, we chose poly(ethylene oxide) with $M_{n}=5000$ with a low dispersity of 1.1 as the hydrophilic block because it would serve as the minority domain in the amphiphilic copolymers. After trityl ether group was successfully introduced into PEG chain end, the vinyl group on trityl was converted to hydroxy group in the presence of 9-BBN in THF to afford hydroxy trityl ether end functionalized PEG (PEG-HydroxyTTE). The disappearance of vinyl signals from proton NMR was used to monitor the reaction. Finally, trityl ether end functionalized ATRP macroinitiator PEG-TTE-Br was prepared by treatment of PEG-HydroxyTTE with 2-bromoisobutyryl bromide in the presence of triethylamine in THF. The chain end functionality was determined to be more than $95 \%$ by the ${ }^{1} \mathrm{H}$ NMR analysis by comparing the integrals of methoxy end group of PEG at $3.37 \mathrm{ppm}$ to that of two new resonances at $1.90 \mathrm{ppm}$ and $4.29 \mathrm{ppm}$, which correspond to methyl protons adjacent to bromo atom and methylene protons adjacent to ester group, respectively (see Figure 1). Meanwhile, the appearance of a new absorption band at around $1730 \mathrm{~cm}^{-1}$ in FT-IR spectrum also verified the formation of the ester carbonyl group.

Then this trityl ether end functionalized macroinitiator PEG-TTE-Br was used to initiate MMA monomer under typical ATRP conditions with anisole as solvent and $\mathrm{CuCl}$ and hexamethylenetetramine as catalyst and ligand. Two copolymerizations were conducted using different ratio of monomer/initiator and were found to be able to proceed very smoothly (Scheme 2). In our study, the molecular weights of MMA blocks were designed to be in the region of 50,000 to 90,000 Da in order to fabricate thin films with different domain size. Good control of molecular weights and low dispersity between 1.18-1.25 were obtained for these two copolymers by ${ }^{1} \mathrm{H}$ NMR and GPC analysis (Figure 2 and Figures S1, S2, S5 and S6 in Supplementary material). This methodology has also been proven to be versatile to a wide range of methacrylate type of monomers. Copolymerizations of PEG-TTE-Br with other methacrylate monomers bearing diverse ester substituent have also been carried out successfully, such as n-butyl methacrylate, trimethylsiloxy-2-ethyl methacrylate, 11-[4-(4'-butylphenylazo)phenoxy]-undecyl methacrylate and 6-(4'-cyanobiphenyl-4-yloxy)hexyl methacrylate.

The resulted two diblock copolymers exhibit same solubility as PMMA since PMMA acts as majority component in the copolymers. Generally, they have very good solubility in chloroform, toluene, THF and DMF. Differential scanning calorimetry (DSC) analysis shows that only a singular glass transition temperature $\left(\mathrm{T}_{\mathrm{g}}\right)$ is seen for the block copolymers at $104.6^{\circ} \mathrm{C}$ for Polymer 1 and $112.3^{\circ} \mathrm{C}$ for Polymer 2 (see Figures $\mathrm{S} 3$ and $\mathrm{S} 4$ in Supplementary material). There was a slight increase of $8 \mathrm{~K}$ in Tg when PEG fraction in copolymers 
(A)<smiles>COCCOCCOS(C)(=O)=O</smiles>

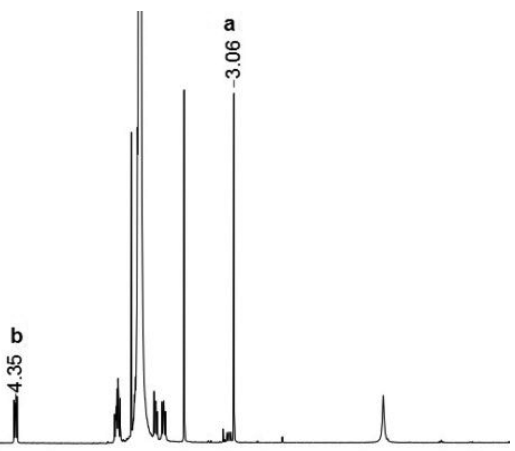

(B)
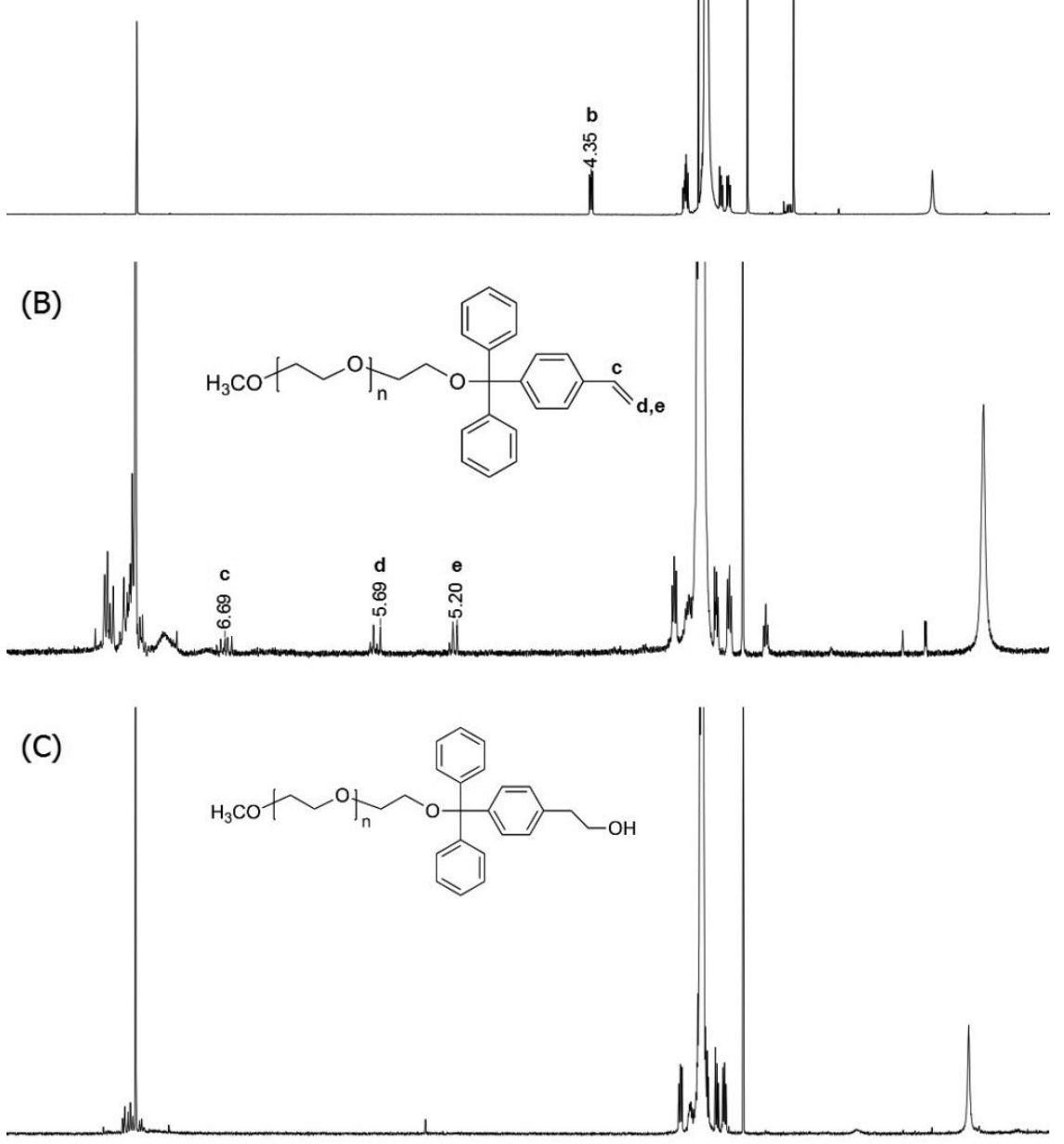

(D)
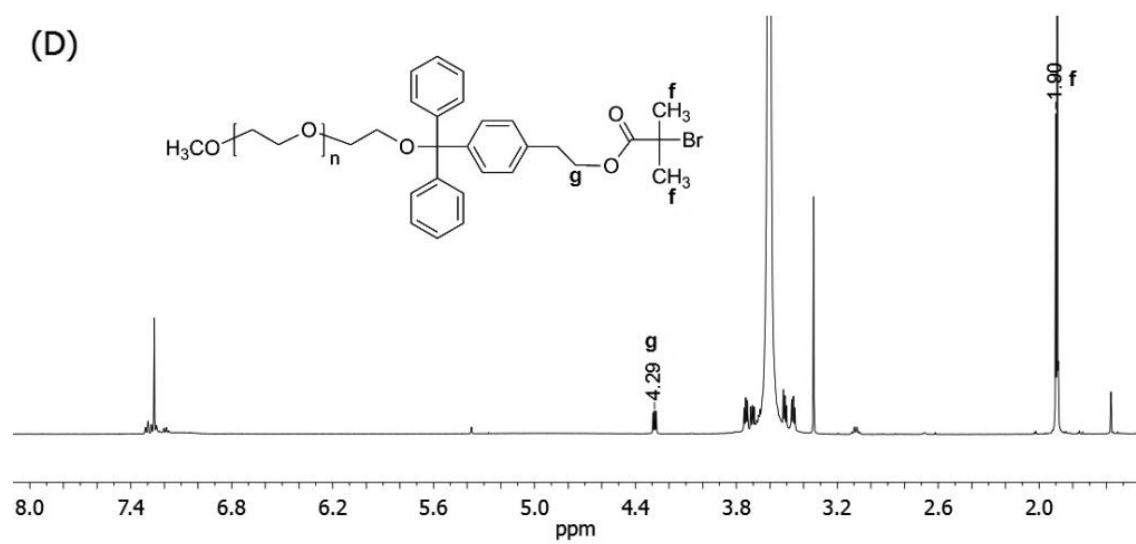

Figure 1: ${ }^{1} \mathrm{H}$ NMR spectra of the synthesis of ATRP macroinitiator: (a) MPEG-mesylate, (b) PEG-VinylTTE, (c) PEG-HydroxyTTE, (d) PEG-TTE-Br.

decreased from 8.3\% for Polymer 1 to 5.5\% for Polymer 2, which can be estimated using Fox equation for miscible phases. The absence of melting point of PEG block together with the observation that there is no discernable phase separation in as-prepared thin films (Figures $4 \mathrm{~d}$ and 5d) indicates that two blocks of copolymers are indeed miscible. The characterization results are summarized and reported in Table 1. 
<smiles>C=C(C)C(=O)OCC(=O)OCCc1ccc(C(OCCOCCCOC)(c2ccccc2)c2ccccc2)cc1</smiles><smiles>COCCOCCCOC(c1ccccc1)(c1ccccc1)c1ccc(CCOC(=O)C(C)(C)C(C)(C)CC(C)(C(=O)OC)C(C)C)cc1</smiles>

Scheme 2: Synthesis of PEG-b-PMMA acid cleavable diblock copolymers using ATRP method.

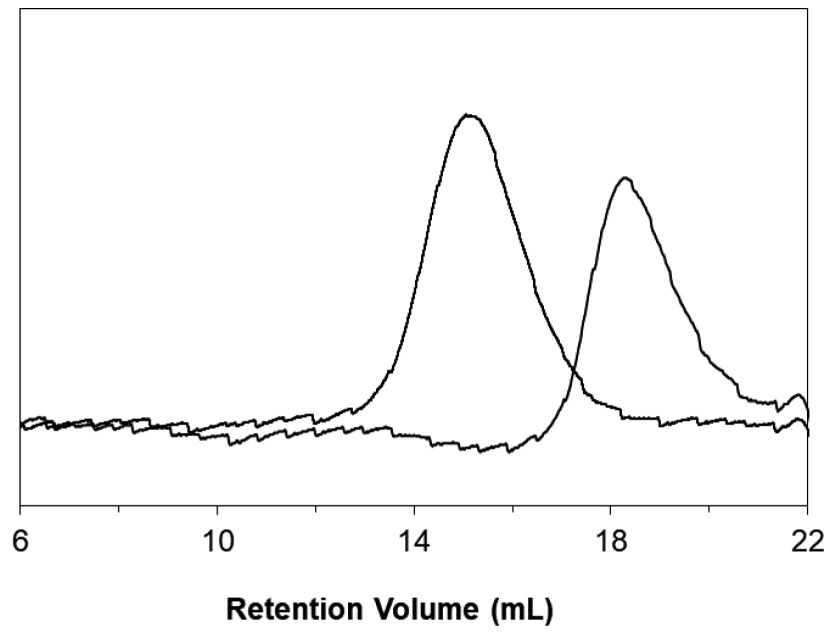

Figure 2: SEC curves for ATRP macroinitiator PEG-TTE-Br (right curve) and diblock copolymer Polymer 2 (left curve).

As demonstrated above, two amphiphilic poly(ethylene glycol)-block-poly(methyl methacrylate)s featuring trityl ether group at junction were successfully prepared. However, to apply such materials in nanoporous thin films, the selective removal of one domain under mild and technologically compatible conditions has to be achieved. To examine the degradation of the block copolymers at the junction under acidic conditions, Polymer 2 was dissolved in toluene and treated with trifluoracetic acid for about $20 \mathrm{~min}$ at room temperature. After washing with water, ${ }^{1} \mathrm{H}$ NMR of the resultant residue showed that signal peaks were only attributable to PMMA, as shown in Figure 3. GPC analysis of residue also indicated that the block copolymer was completely cleaved at the trityl ether junction under this mild acidic
Table 1: Characterization results of acid cleavable PEG- $b$-PMMA diblock copolymers.

\begin{tabular}{|c|c|c|c|c|}
\hline Polymer & $\begin{array}{c}M_{\mathrm{n}} \text {, of MMA } \\
\text { block } \\
(\mathrm{g} / \mathrm{mol})^{\mathrm{a}}\end{array}$ & $\begin{array}{c}M_{w} / M_{n} \text { of } \\
\text { diblock } \\
\text { copolymers }^{b}\end{array}$ & $\begin{array}{c}\mathrm{T}_{\mathrm{m}} \text { of } \\
\text { PEG } \\
\text { block }^{\mathrm{c}}\end{array}$ & $\begin{array}{l}T_{g} \text { of } \\
\text { PMMA } \\
\text { block }^{e}\end{array}$ \\
\hline Polymer 1 & 55000 & 1.25 & $N D^{d}$ & $104.6^{\circ} \mathrm{C}$ \\
\hline Polymer 2 & 85000 & 1.18 & $N D^{d}$ & $112.3^{\circ} \mathrm{C}$ \\
\hline \multicolumn{5}{|c|}{$\begin{array}{l}\text { a Calculated from }{ }^{1} \mathrm{H} \text { NMR of block copolymer. } \\
{ }^{\mathrm{b}} \text { Molecular weight distribution determined from SEC. } \\
{ }^{\mathrm{C}} \text { Melting point determined by differential scanning calorimetry. } \\
{ }^{\mathrm{d}} \text { Not detected. } \\
\text { e Glass transition temperature determined by differential scanning } \\
\text { calorimetry. }\end{array}$} \\
\hline
\end{tabular}

condition because molecular weight of the residue was consistent with molecular weight of the MMA block of copolymer Polymer 2 (see Scheme 3). A similar behavior was observed for Polymer 1 as well after treating with TFA in solution at room temperature. Both PEG and PMMAs blocks have shown excellent stability to the acidic condition from NMR analysis, while cleavage occurs readily only at junction of the block copolymers.

To verify the acid cleavage property of the polymers in thin film morphology, the topography and the phase distribution was studied using AFM at each stage of fabrication. Figure 4 shows the AFM height (Figures 4a-c) and phase (Figures $4 \mathrm{~d}-\mathrm{f}$ ) image at each stage for Polymer 1. The as-coated films are in a quenched-disordered state, trapped in a high-energy configuration (Figures $4 \mathrm{a}$ and $4 d)$. Figures $4 \mathrm{~b}$ and $4 \mathrm{e}$ represent the morphology after annealing. The solvents used for DIA were selected based 
on the Hansen solubility parameters. Here the PEO and PMMA are insoluble in heptane while for toluene, PEO is classified as soluble and PMMA is almost soluble (36). Also, toluene and n-heptane are completely miscible to form a homogenous mixture. It is seen that there is a little signature of phase separation after annealing, which becomes more prominent after the acid vapour treatment

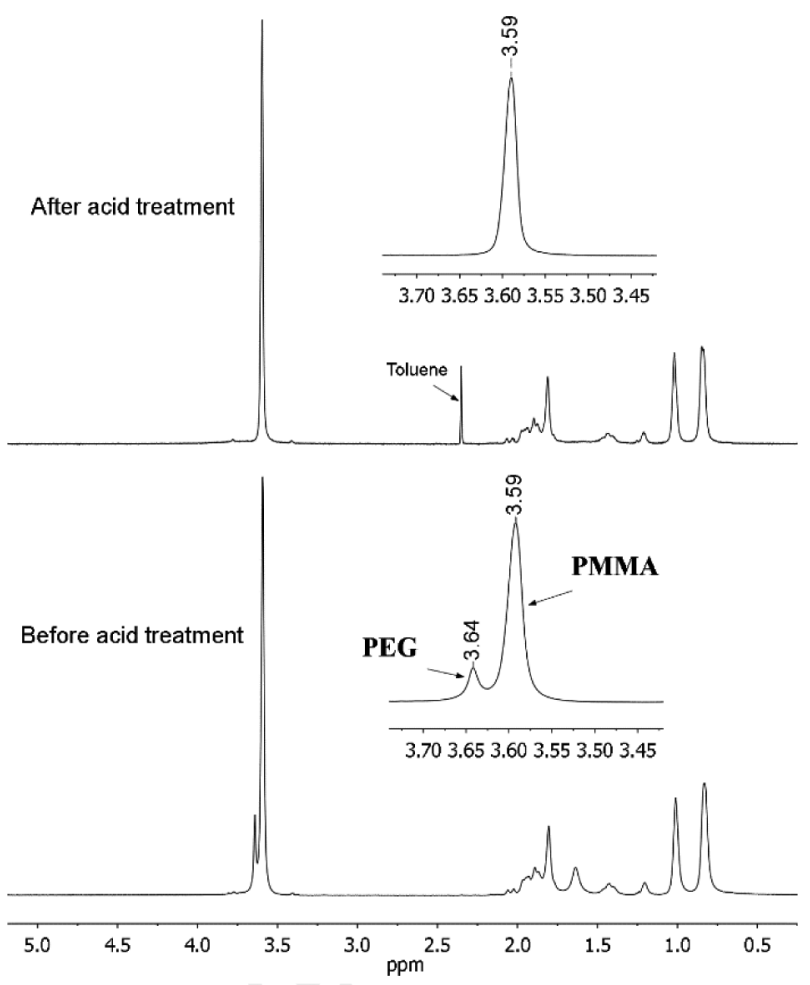

Figure 3: ${ }^{1 H}$ NMR spectra of diblock copolymer Polymer 2 before and after acid treatment using TFA. and washing (Figures 4c and 4f). The phase separation causes the formation of vertically aligned cylinders of PEO embedded within the matrix of PMMA. The acid vapour treatment causes the cleavage of PEO domains at the trityl ether junction, which can then remove effectively by washing with methanol/DI water to generate nano porosity. Figure 5 (a,d; b,e; c,f) represents the AFM height and phase image of as-coated Polymer 2, after DIA and after acid vapour treatment with TFA followed by washing respectively.

To further efficiently study the order and orientation of pores, the porosity was reconstructed from AFM height images using image analysis without affecting the relative positions of the pore. Figures $6 \mathrm{a}$ and $6 \mathrm{~d}$ show the threshold images of Figures $4 \mathrm{c}$ and $5 \mathrm{c}$, respectively, which were used for the calculation of average for pore size, pore size distribution and pore density. The pore size and its density were found by the particle size analysis tool of the ImageJ software, and the same was used for the calculation of pore size distribution which was fitted to Gaussian curve. The average pore size and pore density were found to be $\sim 6.7 \mathrm{~nm}$ and 8352 pores $/ \mu \mathrm{m}^{2}$ for Polymer 1 (Figure 6a) whereas $\sim 5 \mathrm{~nm}$ and 12636 pores $/ \mu \mathrm{m}^{2}$ for Polymer 2 (Figure 6d), respectively.

Later, the threshold images were converted to binary for the porosity reconstruction. Figures $6 \mathrm{~b}$ and $6 \mathrm{e}$ confirm the effective reconstruction where the green channel represents the reconstructed pores and the red channel represents the original threshold images. It is to be noted that the reconstructed image represents only the relative pore positions and not the actual pore size. The fast Fourier transform (FFT) of reconstructed images was used to study the orientation and order of the vertically

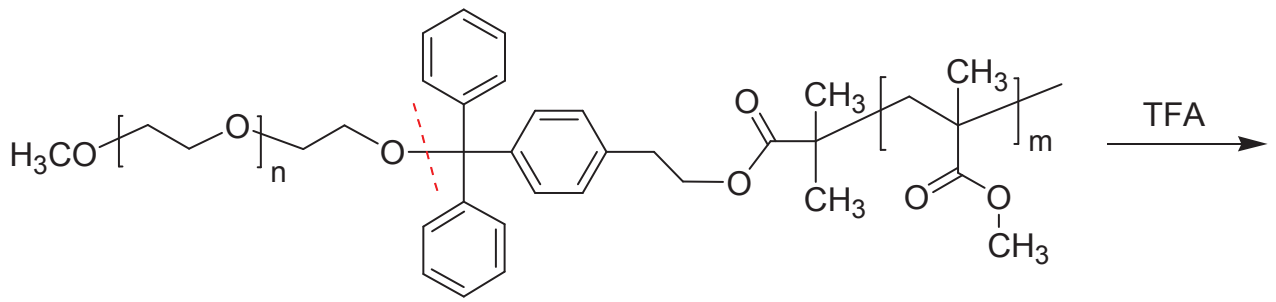<smiles>COC(=O)C(C)(CC(C)(C)C(C)(C)C(=O)OCCc1ccc(C(c2ccccc2)c2ccccc2)cc1)C(C)(C)C</smiles>

Scheme 3: Acid treatment of PEG-b-PMMA (Polymer 2) using TFA in toluene. 

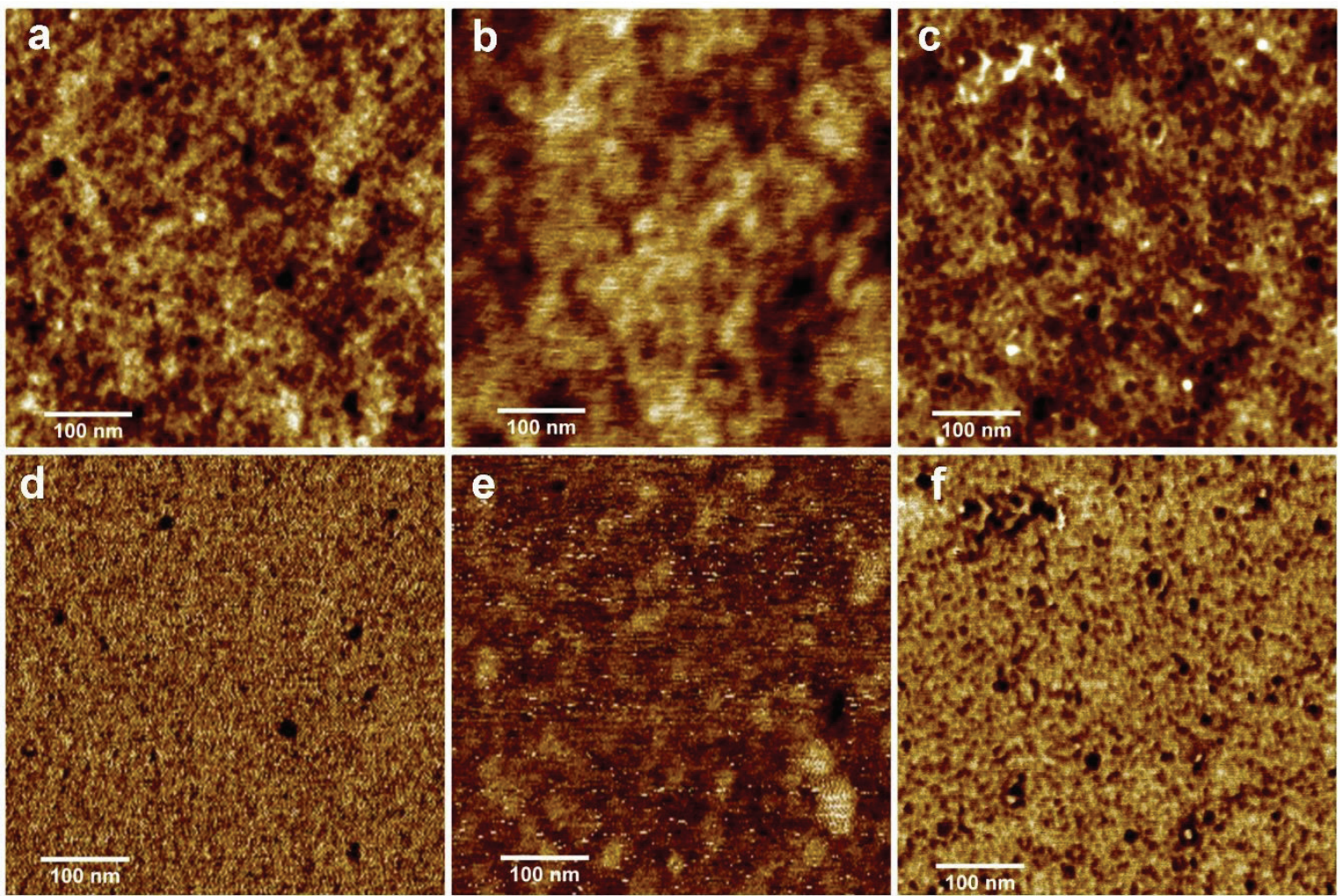

Figure 4: AFM height (a-c) and phase (d-f) of as-casted film (a,d), after DIA (b,e) and after acid vapor treatment followed by washing (c,f) for Polymer 1.
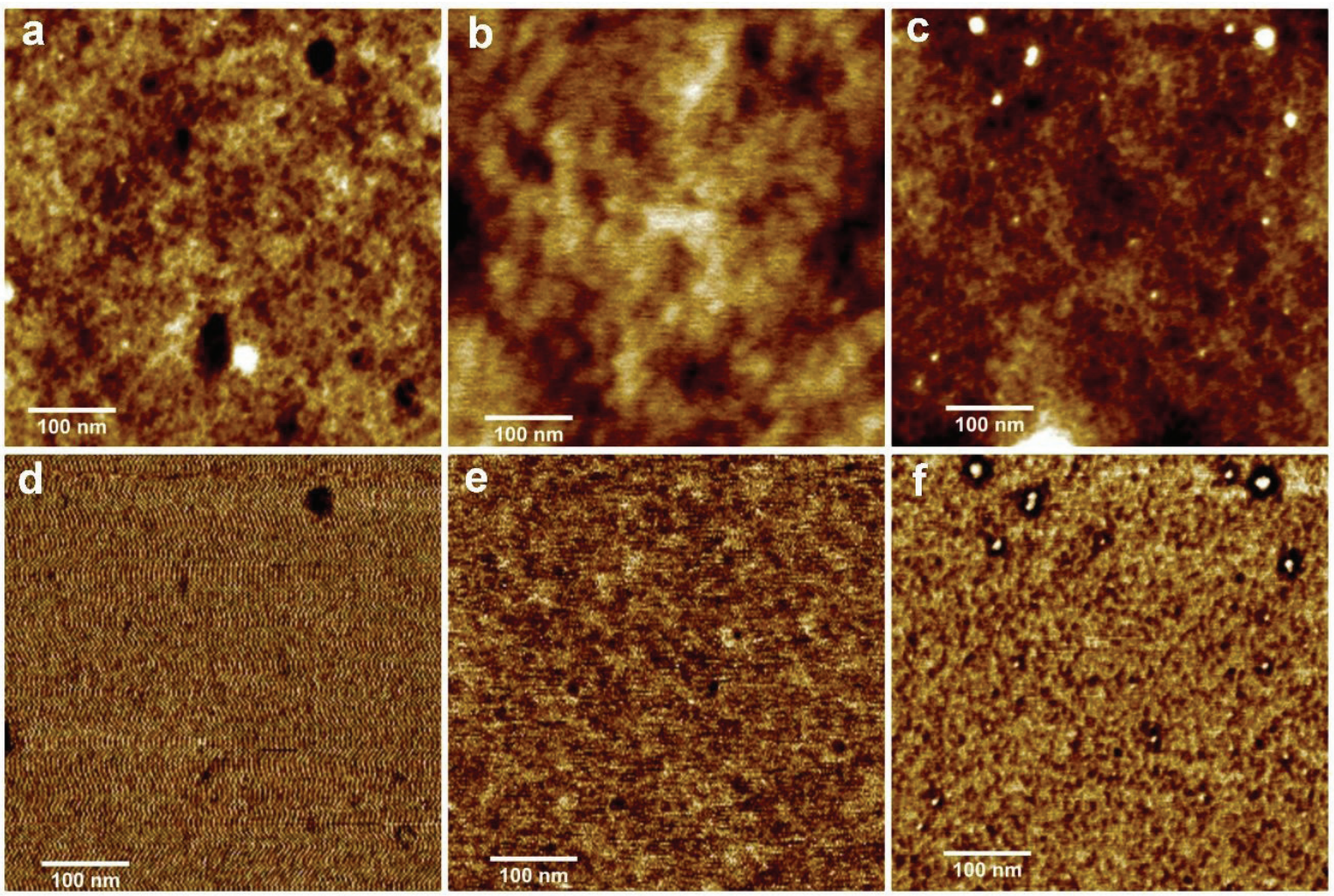

Figure 5: AFM height (a-c) and phase (d-f) of as-casted film (a,d), after DIA (b,e) and after acid vapor treatment followed by washing (c,f) for Polymer 2. 

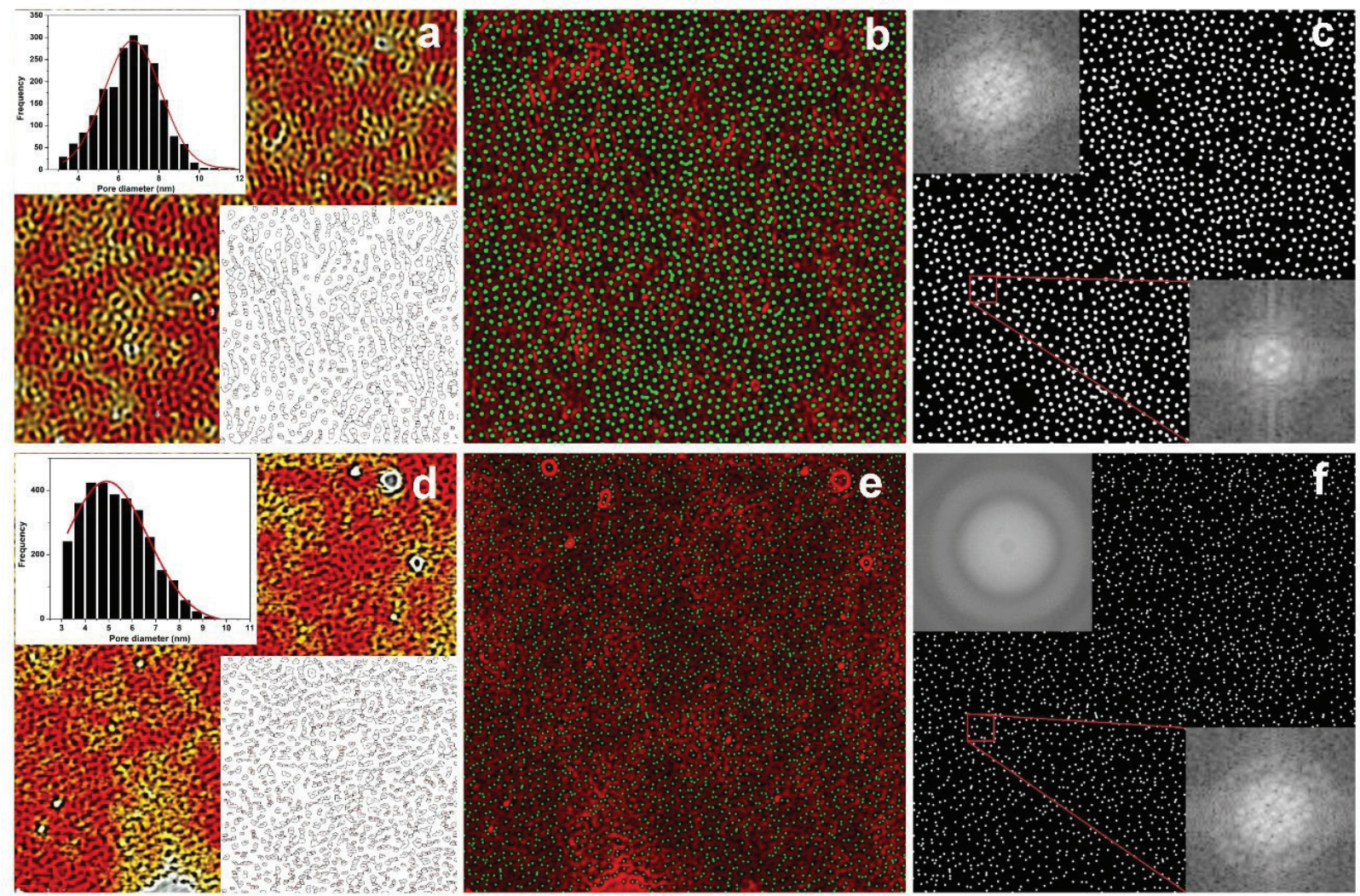

Figure 6: Threshold of AFM height image after acid vapor treatment for Polymer 1 (a) and Polymer 2 (d) inset represents the calculation of pore size and pore size distribution. Composite image of the threshold image (red channel) and reconstructed pores (green channel) for Polymer 1 (b) and Polymer 2 (e), respectively. Reconstructed pores for Polymer 1 (c) and Polymer 2 (f), respectively, inset shows the local $(30 \times 30 \mathrm{~nm})$ and global $(500 \times 500 \mathrm{~nm})$ FFT's.

aligned cylindrical pores. To study the local orientation, the FFT was performed at a $30 \times 30 \mathrm{~nm}$ area whereas for the long-range order it was performed at $500 \times 500 \mathrm{~nm}$ area. It is observed that for Polymer 1, the local FFT displays six prominent spots around the central bright spot, which indicate a hexagonally packed vertical cylinder morphology (Figure 6c inset). The layering distance between the cylindrical rows (L) was found out to be $10 \mathrm{~nm}$ from reciprocal space image and the inter cylinder repeat period $\left(\mathrm{L}_{\mathrm{o}}\right)$ is calculated as $11.55 \mathrm{~nm}$ using $\mathrm{L}_{0}=(2 / \sqrt{3}) \mathrm{L}$. However, the FFT of larger area $(500 \times 500 \mathrm{~nm})$ showed several bright spots around the central bright spot. This indicated a poor long-range order. Similarly, for Polymer 2, the local FFT showed several bright spots and the larger area FFT displayed a ring pattern around the central bright spot indicating a lack of any preferred orientation. The lack of orientation with a smaller pore size and higher pore density for Polymer 2 when compared to Polymer 1 is probably due to the slow diffusion of the higher molecular weight of the matrix component (PMMA) (27). A further study required to achieve a long-range order for Polymer 1 and a preferred orientation with long rage order for Polymer 2 by optimizing the processing time and the solvent/ non-solvent concentration along with the kinetics of the same is currently under progress.

\section{Conclusions}

Two PEO-b-PMMA amphiphilic block copolymers containing trityl ether at junction have been successfully prepared by ATRP polymerization with a good control of molecular weight and dispersity. The key design of these unique copolymers was to introduce an acid cleavable linking group, i.e., trityl ether group into the junction of the block copolymers, which allows the fabrication of highly ordered nanoporous thin films. By acid treatment and subsequent washing with water, the trityl ether linkage at the junctions between two blocks can be readily cleaved and PEG block can be completely removed. Nanoporous thin films have been obtained with the pore size of 5-7 $\mathrm{nm}$. More studies on morphology of thin films and optimization of thin film forming conditions and cleavage conditions of PEO minority block are undergoing. 


\section{References}

1. Chen Q., Luo M., Hammershøj P., Zhou D., Han Y., Laursen B.W., et al., Microporous polycarbazole with high specific surface area for gas storage and separation. J. Am. Chem. Soc., 2012, 134, 6084-6087.

2. Wood C.D., Tan B., Trewin A., Su F., Rosseinsky M.J., Bradshaw D., et al., Microporous organic polymers for methane storage. Adv. Mater., 2008, 20, 1916-1921.

3. Furukawa H., Yaghi O.M., Storage of hydrogen, methane, and carbon dioxide in highly porous covalent organic frameworks for clean energy applications. J. Am. Chem. Soc., 2009, 131, 8875-8883.

4. Turner J.L., Chen Z., Wooley K.L., Regiochemical functionalization of a nanoscale cage-like structure: robust core-shell nanostructures crafted as vessels for selective uptake and release of small and large guests. J. Control. Release, 2005, 109, 189-202.

5. Liu X., Basu A., Core functionalization of hollow polymer nanocapsules. J. Am. Chem. Soc., 2009, 131, 5718-5719.

6. Li G., Yang X., Wang B., Wang J., Yang X., Monodisperse temperature-responsive hollow polymer microspheres: Synthesis, characterization and biological application. Polymer, 2008, 49, 3436-3443.

7. Binauld S., Scarano W., Stenzel M.H., PH-triggered release of platinum drugs conjugated to micelles via an acid-cleavable linker. Macromolecules, 2012, 45, 6989-6999.

8. Hu X., Li G., Li M., Huang J., Li Y., Gao Y., et al., Ultrasensitive specific stimulant assay based on molecularly imprinted photonic hydrogels. Adv. Funct. Mater., 2008, 18, 575-583.

9. Kim K.T., Cornelissen J.J.L.M., Nolte R.J.M., van Hest J.C.M., A Polymersome nanoreactor with controllable permeability induced by stimuli-responsive block copolymers. Adv. Mater., 2009, 21, 2787-2791.

10. Yan Q., Zhao Y., Polymeric microtubules that breathe: $\mathrm{CO}_{2}$-driven polymer controlled-self-assembly and shape transformation. Angew. Chem. Int. Ed., 2013, 52, 9948-9951.

11. Lee J.S., Hirao A., Nakahama S., Polymerization of monomers containing functional silyl groups. 5. Synthesis of new porous membranes with functional groups. Macromolecules, 1988, 21, 274-276.

12. Thurn-Albrecht T., Steiner R., Stafford D.C.M., Huang E., Bal M., Tuominen M., et al., Nanoscopic templates from oriented block copolymer films. Adv. Mater., 2000, 12, 787-791.

13. Leiston-Belanger J.M., Russell T.P., Drochenmuller E., Hawker C.J., A thermal and manufacturable approach to stabilized diblock copolymer templates. Macromolecules, 2005, 38, 7676-7683.

14. Zhao H., Gu W., Sterner E., Russell T.P., Coughlin E.B., Theato P., Highly ordered nanoporous thin films from photocleavable block copolymers. Macromolecules, 2011, 44, 6433-6440.

15. Kang M., Moon B., Synthesis of photocleavable poly(styreneblock-ethylene oxide) and its self-assembly into nanoporous thin films. Macromolecules, 2009, 42, 455-458.

16. Bertrand O., Poggi E., Gohy J., Fustin C., Functionalized stimuliresponsive nanocages from photocleavable block copolymers. Macromolecules, 2014, 47, 183-190.

17. Zhao H., Gu W., Thielke M.W., Sterner E., Tsai T., Russell T.P., et al., Functionalized nanoporous thin films and fibers from photocleavable block copolymers featuring activated esters. Macromolecules, 2013, 46, 5195-5201.

18. Klaikherd A., Ghosh S., Thayumanavan S., A facile method for the synthesis of cleavable block copolymers from ATRP-based homopolymers. Macromolecules, 2007, 40, 8518-8520.

19. Zhang M., Yang L., Yurt S., Misner M.J., Chen J., Coughlin E.B., et al., Highly ordered nanoporous thin films from cleavable polystyrene-block-poly(ethylene oxide). Adv. Mater., 2007, 19, 1571-1576.

20. Yurt S., Anyanwu U.K., Scheintaub J.R., Coughlin E.B., Venkataraman D., Scission of diblock copolymers into their constituent blocks. Macromolecules, 2006, 39, 1670-1672.

21. Sivanandan K., Chatterjee T., Treat N., Kramer E.J., Hawker C.J., High surface area poly(3-hexylthiophenes) thin films from cleavable graft copolymers. Macromolecules, 2010, 43, 233-241.

22. Shchepinov M.S., Korshun V.A., Recent applications of bifunctional trityl groups. Chem. Soc. Rev., 2003, 32, 170-180.

23. Grubbs R.B., Nitroxide-mediated radical polymerization: limitations and versatility. Polym. Rev., 2011, 51, 104-137.

24. Sinturel C., Vayer M., Morris M., Hillmyer M.A., Solvent vapor annealing of block polymer thin films. Macromolecules, 2013, 46, 5399-5415.

25. Majewski P.W., Yager K.G., Rapid ordering of block copolymer thin films. J. Phys. Condens. Matter., 2016, 28, 403002.

26. Albert J.N., Epps III T.H., Self-assembly of block copolymer thin films. Mater. Today, 2010, 13, 24-33.

27. Kim E., Ahn H., Park S., Lee H., Lee M., Lee S., et al., Directed assembly of high molecular weight block copolymers: highly ordered line patterns of perpendicularly oriented lamellae with large periods. ACS Nano, 2013, 7, 1952-1960.

28. Seguini G., Zanenga F., Giammaria T.J., Ceresoli M., Sparnacci K., Antonioli D., et al., Enhanced lateral ordering in cylinder forming PS-b-PMMA block copolymers exploiting the entrapped solvent. ACS Appl. Mater. Inter., 2016, 8, 8280-8288.

29. Peng J., Xuan Y., Wang H., Li B., Han Y., Solvent vapor induced dewetting in diblock copolymer thin films. Polymer, 2005, 46, 5767-5772.

30. Peng J., Kim D.H., Knoll W., Xuan Y., Li B., Han Y., Morphologies in solvent-annealed thin films of symmetric diblock copolymer. J. Chem. Phys., 2006, 125, 064702.

31. Kim K., Park S., Kim Y., Bang J., Park C., Ryu D.Y., Optimized solvent vapor annealing for long-range perpendicular lamellae in PS-b-PMMA films. Macromolecules, 2016, 49, 1722-1730.

32. Modi A., Bhaway S.M., Vogt B.D., Douglas J.F., Al-Enizi A., Elzatahry A., et al., Direct immersion annealing of thin block copolymer films. ACS Appl. Mater. Inter., 2015, 7, 21639-21645.

33. Park W.I., Kim J.M., Jeong J.W., Jung Y.S., Deep-nanoscale pattern engineering by immersion-induced self-assembly. ACS Nano, 2014, 8, 10009-10018.

34. Longanecker M., Modi A., Dobrynin A., Kim S., Yuan G., Jones R., et al., Reduced domain size and interfacial width in fast ordering nanofilled block copolymer films by direct immersion annealing. Macromolecules, 2016, 49, 8563-8571.

35. Zhao X., Janda K.D., Syntheses of alkylated malonates on a traceless linker derived soluble polymer support. Tetrahedron Lett., 1997, 38 5437-5440.

36. Hansen C.M., Hansen solubility parameters: A user's handbook. CRC Press, 2002. 